\title{
Voltage Unbalance Reduction of a Local Transformer Area with Domestic Asymmetrical Inverter and Optimal Control Design
}

\author{
László Neukirchner *, Attila Göllei, Péter Görbe, Attila Magyar \\ Department of Electrical Engineering and Information Systems, Faculty of Information Technology, University of \\ Pannonia, Egyetem street 10. H-8200, Veszprém, Hungary
}

\begin{abstract}
The three-phase unbalance problem can introduce additional losses in distribution networks due to both negative and zero sequence components, which leads to inefficient power consumption and increased $\mathrm{CO}_{2}$ emission of low voltage transformer area, moreover it causes safety possible malfunction of energy transportation networks. The aim of this paper is to introduce a new voltage unbalance indicator and a control algorithm that compensates source voltage unbalances. To examine the available measures of voltage asymmetry and make a comparison from the transient behaviours point of view. Analytical and simulation, results are presented to illustrate the effectiveness of the new techniques.
\end{abstract}

\section{Motivation}

By introducing sustainability in domestic power distribution systems, power quality became a more important task to maintain. In Hungary most of the household loads are single phase devices (for example lighting goods, household appliances as washing machines, irons, televisions, personal computers etc.), and with the exception of some optimal case, they are not evenly distributed between the phases. Furthermore, there is a considerable noise generated by stochastic switching appliances. This causes stochastic disturbing unbalance in the load currents which cases unbalances load of the low voltage transformer, and causes amplitude and phase unbalance in the voltage phasor trough the serial impedance of the low voltage transportation line wires and connecting devices. It should be see clearly, that the unbalance situation further exacerbated by using single phase grid tie inverter systems in the size of typical small household domestic power plants (SHDPP) (1 - 5 kW) and the produced electrical power from renewable power source, wind and solar, stochastic behavior too.
In this paper a solution is purposed to reverse this phenomena, by using renewable sources, to not increase, but to compensate the disturbances on the network. Manufacturers currently being introduced their three phase grid synchronized inverters in this size to the market to lower the unbalance caused the spare power asymmetrical current injection to the low voltage grid. The uniform distribution of loads and generators can lower this effect, but the limited number of households in one transformer area is unable to statistically equalize the stochastic behavior of loads and sources. Rising number of SHDPP can amplify these disturbances. This unbalance cause suboptimal operation of low voltage three phase transformers to create undesirable additional power loss and $\mathrm{CO}_{2}$ emission rising, to increase the calculated carbon footprint of an average household and increase in the probability of malfunction of low voltage energy transportation system.

${ }^{*}$ Corresponding author

E-mail: neukirchner.laszlo@ virt.uni-pannon.hu

(C) 2016 International Association for Sharing Knowledge and Sustainability

DOI: 10.5383/ijtee.12.02.009 


\section{Regulated Indicators of Voltage Asymmetry}

In a three-phase system, voltage unbalance takes place when the magnitudes of phase or line voltages are different and the phase angles differ from the balanced conditions, or both. These definitions have important implications when studying for example, the effects of voltage unbalance on the performance of three-phase induction machines. Although on the meaning of the term voltage unbalance, where different standards introduce different conventional definitions as follows $\mathrm{Eq}(1)$ :

$$
\begin{aligned}
& V_{936}=\frac{\max \left\{V_{a n}, V_{b n} V_{c n}\right\}-\min \left\{V_{a n} V_{b n} V_{c n}\right\}}{\operatorname{mean}\left\{V_{a n} V_{b n} V_{c n}\right\}} \times 100 \\
& V_{112}=\frac{\text { max deviation from mean of }\left\{V_{a n}, V_{b n} V_{c n}\right\}}{\operatorname{mean}\left\{V_{a n^{\prime}} V_{b n} V_{c n}\right\}} \times 100 \\
& M D V=\frac{\max \text { deviation from mean of }\left\{V_{a n^{\prime}} V_{b c^{\prime}} V_{c a}\right\}}{\operatorname{mean}\left\{V_{a n^{\prime}} V_{b c^{\prime}} V_{c a}\right\}} \times 100 \\
& T D V=\frac{\text { negative sequence voltage }}{\text { positive sequence voltage }} \times 100
\end{aligned}
$$

$V_{a n}, V_{b n}, V_{c n}$

where are the phase-to-neutral voltages, are the line-to-line voltages, is equal to the voltage amplitude of the fundamental frequency and is the voltage amplitude of the n-th harmonic. Although the harmonic distortion (Görbe et al. 2011) does not relate closely to the asymmetry phenomenon, it can cause a variety of problems like dropping efficiency of electric motors. The above four definitions could produce different values for a single cases (current unbalance formulations are simply obtained by replacing voltage with current components). The first two standards ignore the 120 degree phase difference unbalance and only take the amplitudes into account. The last two definitions, MDV and TDV, are sensitive to the phase difference unbalance. Nevertheless, these definitions ignore zero sequence components and harmonic distortion that are always present in three-phase four-wire systems (Tavakoli et al. 2011).

\section{Novel Norm for Measuring Voltage Asymmetry}

As mentioned, in a three-phase system analyzing voltage asymmetries with the help of existing norms raises questions, like the calculating width zero component, or harmonic distortions. It can be stated that every difference between the ideal and the measured voltage in both amplitude phase and sub-harmonics causing a form of asymmetry. In this case the problem can be viewed in a geometrical point of view. The three-phase voltage system's phasor diagram contains three phase-to-neutral voltage vectors which indicate the points of the triangle or three line-to-line vectors which indicate the edges of the triangle (Fig. 1.).

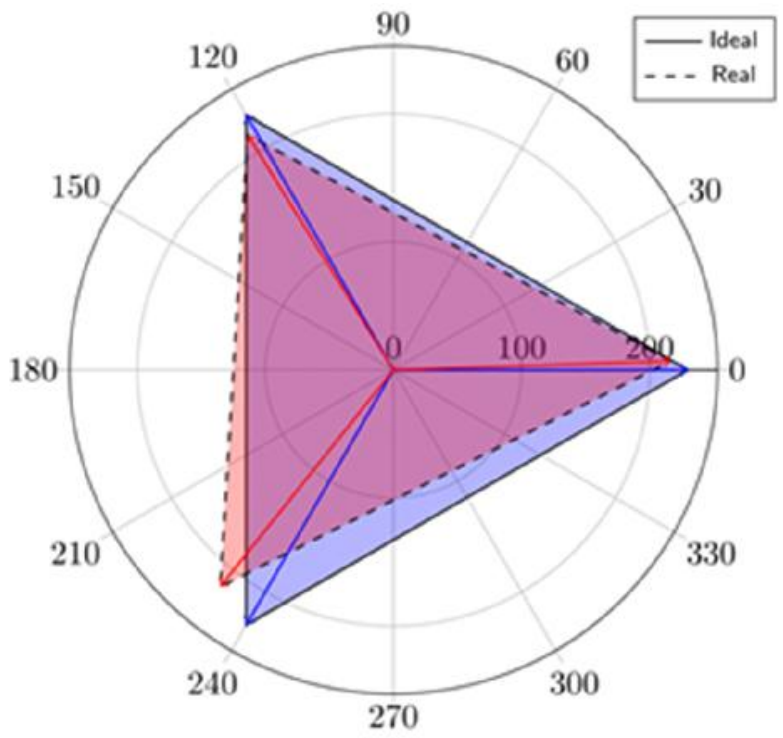

Figure 1: Calculation of Geometrical Norm Displayed on Polar Coordinate System

The two triangles always intersect aside from extreme cases. The control goal is to eliminate the areas of the triangles, where they do not cover each other. This produces a highly cost effective reduction method in the terms of power needed for control. The disadvantage of this method that high amount of calculation capacity compared to other methods and cannot handle distortions alone. The calculation of asymmetry error is given by (4).

\section{$G=$ Area of $\left(\Delta_{\text {idaal }} \cup \Delta_{\text {raal }}-\Delta_{\text {idaal }} \cap \Delta_{\text {raal }}\right)$,}

where ideal indicates the triangle composed of ideal voltage vectors and real indicates the triangle composed of real voltage vectors subtraction of the ideal and the real triangle's union and intersection gives an error indicator of the difference (Fig. 3.).

\section{Asymmetry Compensation Control}

\section{Low Voltage Power Grid Model}

The mathematical model of the nonlinear distorted network was simulated in Matlab Simulink using the Power Electronics Toolbox (see Figure 2). The flow chart of the model consists of a small $400 \mathrm{~V}, 50 \mathrm{~Hz}$ transformer station. Power distribution cable networks were the nonlinearities was implemented with serial resistive and inductive elements as well as capacitive couplings, and domestic load system. Three types of loads were modeled: ohmic loads (representing e.g. heating devices and traditional bulbs, ohmic loads with serial inductances (representing e.g. motors and rotating household appliances) and capacitive input stage loads representing simple nonlinear switching mode power supplies (see Figure 3.). 


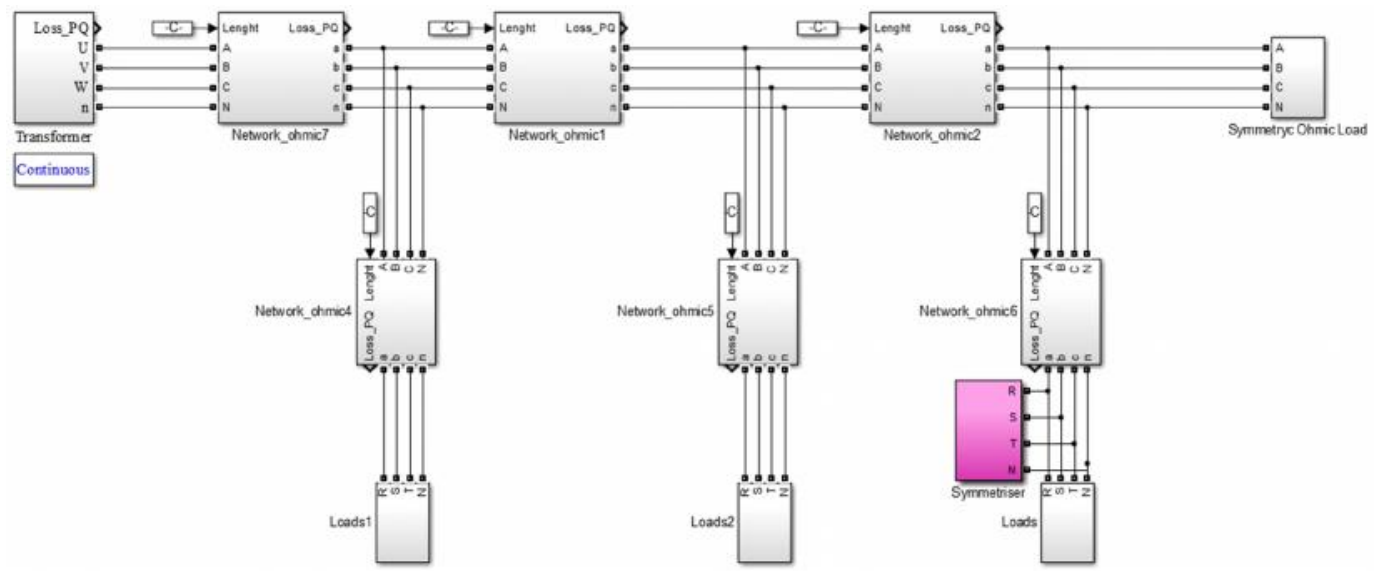

Figure 2: Chart of Low Voltage Three Phase Grid Model

\section{The Controller Structure}

In the real application the grid tie inverters connected to the low voltage network normally cannot sense the exact three phase voltage and current time functions on the transformer secondary connection, but only the voltage time functions at the connection point, behind the power meter of the household. Sensing the needed voltage and current values has technical and legal difficulties. Some of them will be overcome in the near future using smart grid interfaces between the provider and the end user appliances. Now only the voltage measurement is feasible, so a control structure had to be used. We should conclude the actual voltage and current asymmetry of the transformer from the connection point voltage status. The problem is that, the exact mathematical relation is nonlinear because the nonlinear, and highly time variant loads of the network, we should use a control strategy to cope this nonlinear and time variant energy system. For this purpose we chose an asynchronous parallel pattern search method (Asynchronous Parallel Pattern Search, or APPS) which could be able to control our scenario. We applied a variant of the gradient method that is a first-order optimization (minimization) algorithm for a multivariate function $f(x)$. The point $x(k)$ corresponding to the local minimum can be calculated from the negative gradient $\Delta f(x)$, that gives the value and direction of the corresponding step in the parameter space. The next step is made in the direction of gradient with the proper sign. This sequence of steps $\operatorname{Eq}(3)$, ideally, converges to local multivariate extreme value of the function (Snyman et al. 2005).

$$
x(k)=x(k-1)-\mathrm{t}_{\mathrm{k}} \quad \Delta \mathrm{f}(\mathrm{x}(\mathrm{k}-1)) k=1,2, \ldots, n .
$$

The controlled electrical system is described by multivariate non-linear differential equations, the optimization of which is infeasible to derive using the differentiation of an error function. Therefore, the optimization methods based on direct differentiation are not applicable.
In such cases, when high computational power is needed for performing long time-consuming simulations, the APPS method can utilized. The search pattern $P$ is based on the sampling of the error function (selected norm) on a "grid", and it corresponds to variables or subsets of variables in each point in the independent variable or parameter space easily. At the same time, the norm values at these points can be calculated independently (Polyak 1987) Eq(4).

$$
\begin{aligned}
& \text { iff } \Delta k>0, \\
& \left.x(k+1)=x(k)+\Delta k \cdot d_{i}\right) \leq f(x(k)), k=1,2, \ldots, n, \\
& x(k) \in R_{n}
\end{aligned}
$$

where the parameter is, and the search pattern $p \in D=\left\{d_{1}, \ldots d_{n}\right\}$ is taken from a predefined finite set. In this case, the error function values should be calculated for each pattern $p$ in the set $D$. If the error function is not decreasing in any of the directions, then the step size should be reduced (e.g. by half). As the competing directions are different, if there isn't enough computing power available for direction vector $P$, synchronization should not be maintained. In this case we are talking about the asynchronous case (Kolda et al. 2001). In the case of our controller, an individual $p$ vector is defined for each output variable, and the optimization was performed in each direction asynchronously and shifted in time. As being prescribed, the full state-space model was not available in analytic form, and the differential equations describing the system are not linear. Most likely, the error function has a single local minimum as a symmetric amplitude and phase values. Approaching the minimal value of norm, the controller uses adaptive increments that are proportional to the norm itself. Because of the complex interactions between the components of the controller, only one parameter is changed at a time, even if the values of the amplitude and phase components in specific time slot changes. 
The algorithm moves along the six axes of six separate time slots close to the local minimum of the error function. Each time slot is six period $(120 \mathrm{~ms})$ long.

The controller frequently initiates a $20 \mathrm{~ms}$ up and down step function with two relaxation cycles. In each iteration only one physical value is changeling of the three injected current amplitude and three phase values. If the change decreases the cost function (the reference norm's normalized value), the controller holds the new value of amplitude or phase for the controlled current sources (Kolda et al. 2001).

\section{Asymmetrical Inverter Design}

Kirchhoff's 1st law permits only constant zero-sum current time functions injected with this structure.

The proposed design is capable of producing non constant zero-sum currents. The applied structure based on a full bridge
IGBT structure used in single phase current injection. Three different IGBT full bridges were used, connected at the output point, thus the structure has $\mathrm{R}, \mathrm{S}, \mathrm{T}$ and 0 connections. One phase uses the intermediate voltage capacitor, but on the other two phases we have to build isolated DC-DC converters significant current load capacity. Unfortunately it can significantly increase the cost of production of the supposed inverter system (Neukirchner et al. 2015).

\section{Controller Performance}

At the beginning of the simulation we examined the static performance of the proposed controller as a verification of the method.

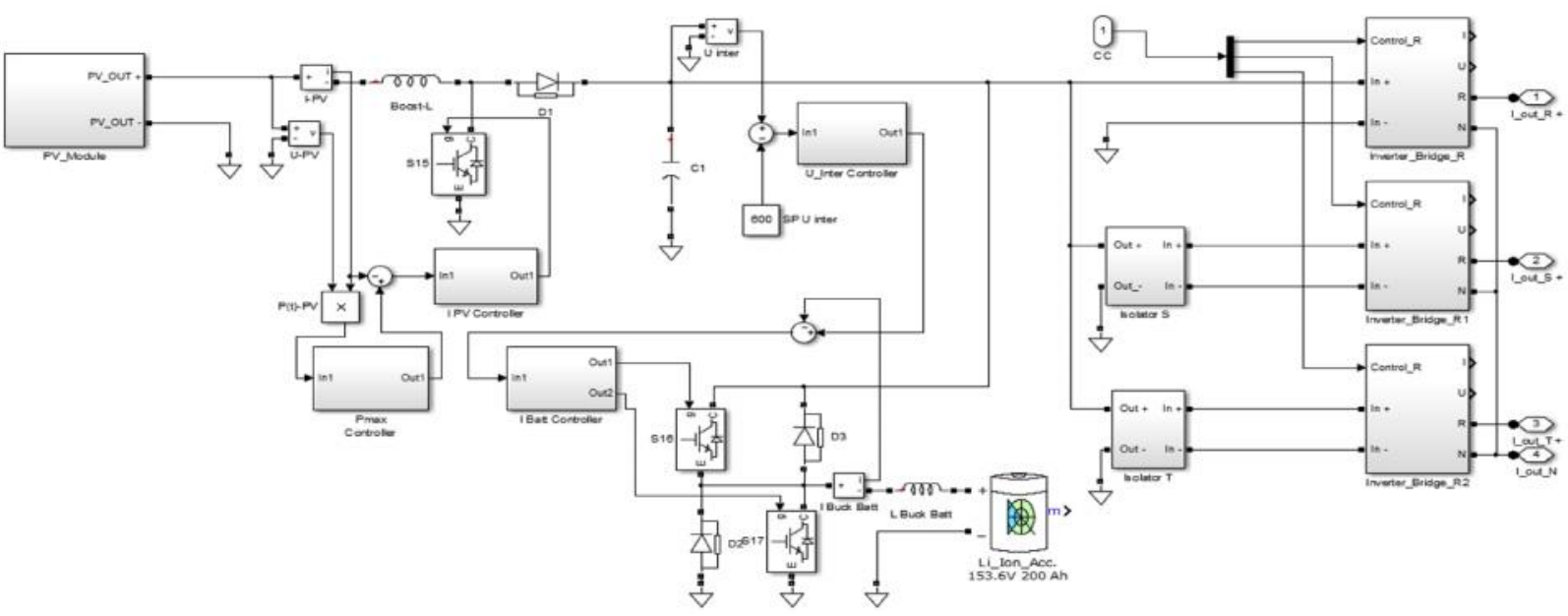

Figure 3: The inverter model, using Matlab/Simulink Powesystems Toolbox. The other standard parts, that the inverter design consists is a standard Maximum Power Point Tracking (MPPT) input stage, an intermediate voltage capacitor with a simple controlled boost converter, a half bridge current controller to charge or recharge the battery pack, an intermediate voltage capacitor, an universal three phase output stage with 3 single phase full bridge IGBT current injector, and 2 high current DC-DC converter.

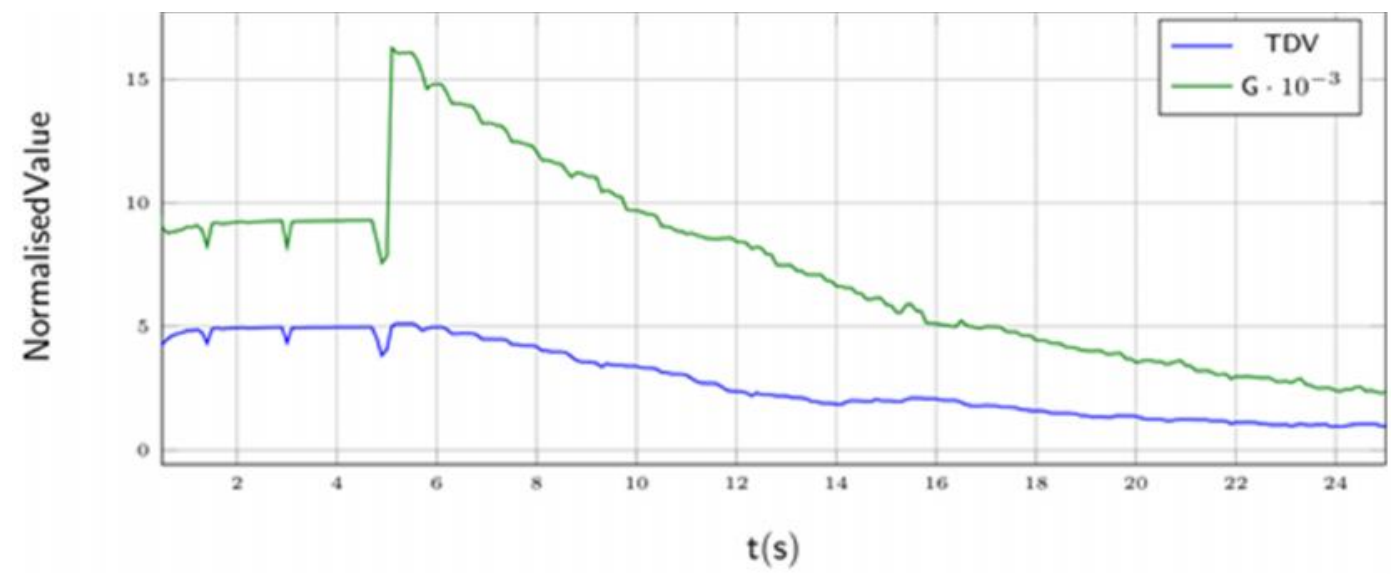

Figure 4: The controller starts at $t=5 \mathrm{~s}$ to operate and compensate the asymmetries via the geometrical asymmetry indicator as cost function. 


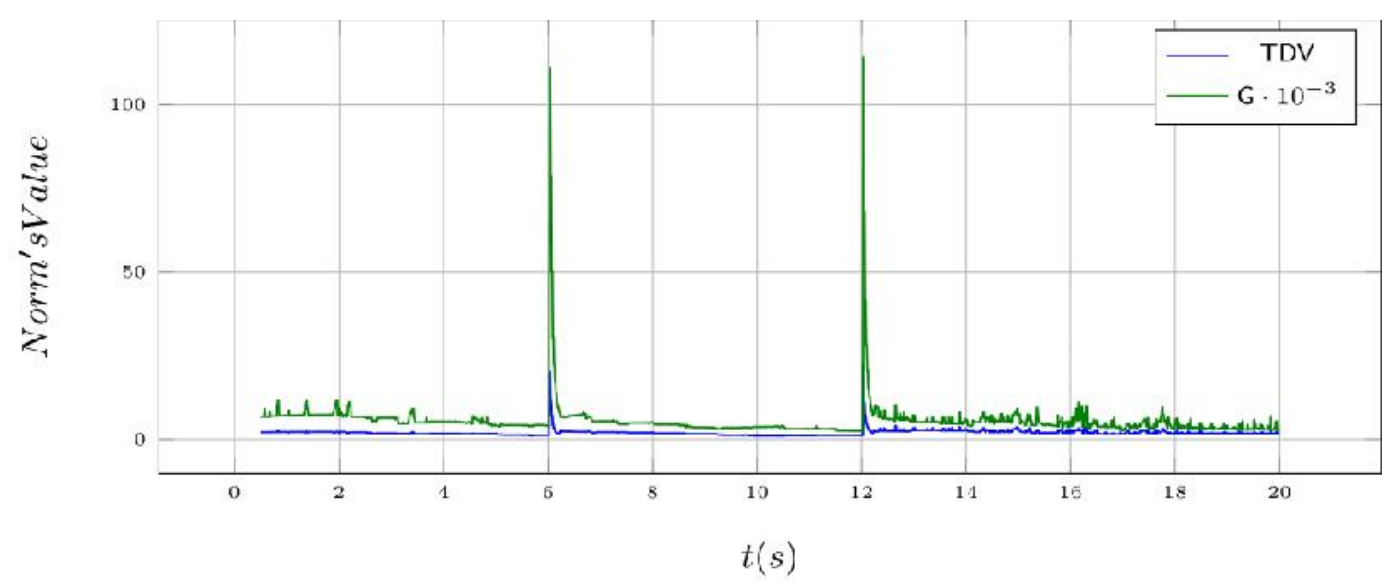

Figure 5: By checking the robustness of the controller, at the $t=6$ the inductive loads switched from the $S$ phase to the $T$, and the capacitive loads form the $\mathrm{T}$ to the $\mathrm{S}$, and at $\mathrm{t}=12$ they switch back to the original state.

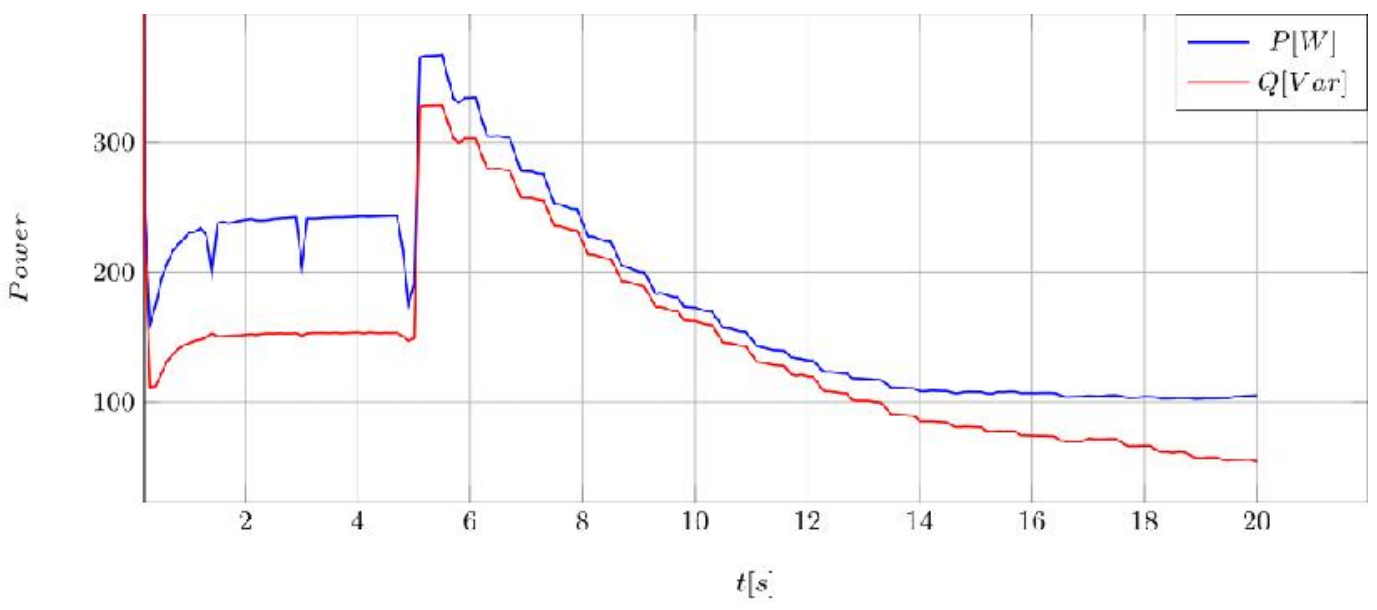

Figure 6: During control high decrease of the network loss was witnessed. At the effective power decreased by 52.21\% and the reactive power by $60 \%$. At $t=5$ the controller switches from regular synchronised symmetrical operation to asymmetry reduction. Note, that the asymmetry values on the network have been set extraordinary high, to demonstrate the capabilities of the controller.

\section{Conclusion}

The currently used measures of voltage asymmetry have been extended with a new norm candidate. The method is more demanding from the computational point of view. An interesting feature of the latter norm is that it checks electrical asymmetry, i.e. the norm of a $120 \%$ rotated version of the ideal tri-phase phasor is zero in the geometrical sense.
The defined norm serves as a cost function in the asymmetry reducing optimal controller structure also presented in the paper. The suggested controller structure enables the residential users owning a domestic power plant to reduce voltage asymmetry measurable at their connection point. 
The fundamental element of the system is a modified three phase asymmetrical inverter design that is capable of injection of any current waveforms to the network. The optimization based control algorithm implies injected waveforms in such a way, that the given asymmetry norm decreases. This optimization problem is usually constrained by the available renewable energy supplied by the power plant. It was possible to decrease the norm value by $96 \%$, together with an overall $52.2 \%$ decrease in effective and $60 \%$ decrease in the reactive power. The fact, that this controller enables the reactive power reduction means, that the power loss, $\mathrm{CO} 2$ emission, i.e. the carbon footprint can also be decreased.

The preliminary results show that this structure can serve as a residential level voltage quality improvement method for the three phase low voltage network.

\section{Acknowledgement}

This contribution has been developed in the framework of the TÁMOP-4.2.2.B-15/1/KONV-2015-0004 Support of scientific groups at University of Pannonia and supported by the Doctoral School of Information Science and Technology of University of Pannonia.

\section{References}

[1] Görbe P., Magyar A., Hangos K.M., 2012, Reduction of power losses with smart grids fuelled with renewable sources and applying EV batteries, Journal of Cleaner Production, vol. 34, 125-137.

[2] Görbe P., Fodor A., Magyar A., Hangos K.M., 2014, Experimental study of the nonlinear distortion caused by domestic power plants, Applied Thermal Engineering, 70(2), 1288-1293.

[3] Neukirchner L., Görbe P., Göllei A., Magyar A., 2015, Carbon Footprint Reduction via Voltage Asymmetry Compensation of Three-Phase Low Voltage Grid Utilizing Small Domestic Power Plants, Chemical Engineering Transactions, vol. 45, 22839216.

[4] Kolda T.G., Hough P.D., Torczon V.J., 2001, Asynchronous parallel pattern search for nonlinear optimization, Society for Industrial and Applied Mathematics: Journal on Scientific Computing, 23(1), 134-156.

[5] Polyak B.T., 1987. Introduction to optimization, Publications Division, New York, USA.

[6] Snyman J. A., 2005. In Practical mathematical optimization: an introduction to basic optimization theory and classical and new gradient-based algorithms. Springer, New York, USA.

[7] Tavakoli Bina M., Kashefi A., 2011. Three-phase unbalance of distribution systems: Complementary analysis and experimental case study, International Journal of Electrical Power \& Energy Systems, 33(4), 817-826. 